\title{
More Elevated Fastballs Associated With Placement on the Injured List due to Shoulder Injury
}

\author{
Austin V. Stone, M.D., Ph.D., Brooks N. Platt, M.D., Brandon S. Collofello, M.D., \\ Aaron D. Sciascia, Ph.D., Timothy L. Uhl, Ph.D., and William B. Kibler, M.D.
}

\begin{abstract}
Purpose: To evaluate whether impending shoulder injury was associated with changes in pitch location or velocity immediately preceding injury. Methods: Pitchers placed on the injured list (IL) due to a shoulder injury between 2015 and 2020 were identified in the Major League Baseball transactions database. Four-seam fastball velocity and frequency of pitch location for each pitch type was collected for each player in the season before placement on the IL and within 1 month of placement on the IL with a minimum of 55 pitches thrown of 1 type. Pitch locations were collected as identified by Baseball Savant's Game-Day Zones. Game-Day Zones were consolidated into high (above the strike zone midpoint) versus low, arm side (closer to the pitcher's arm side of the plate) versus opposite side, and within the strike zone versus out of zone. Repeated measures analysis of variance determined differences in four-seam velocity and the location distribution of 4-seam fastballs, change-ups, and breaking balls among each group. Results: In total, 267 pitchers were placed on the IL for a shoulder injury with the majority diagnosed with inflammation (89/267) followed by strain or sprain (69/267). Four-seam fastball locations significantly increased above the mid-point of the zone $(45.9 \%$ vs $42.4 \%$, $P=.008)$ and out of the strike zone $(48.5 \%$ vs $46.5 \%, P=.011)$ within a month before IL placement. There was no significant change in 4-seam fastball velocity immediately before IL placement. Conclusions: Pitchers threw more elevated 4-seam fastballs and out-of-zone 4-seam fastballs in the month before IL placement for shoulder injury. These findings suggest that a loss of 4-seam fastball command decreases with impending shoulder injury. Level of Evidence: IV, prognostic case series.
\end{abstract}

D espite decades of advanced analytics changing the way baseball is played, injury rates remain high among professional pitchers. The most commonly injured joint in baseball is the shoulder, which

From the Division of Sports Medicine, Department of Orthopaedic Surgery (A.V.S., B.N.P., B.S.C.), and Department of Physical Therapy (T.L.U.), University of Kentucky, Lexington; Department of Exercise and Sport Science, Eastern Kentucky University, Richmond (A.D.S.); and Lexington Clinic Orthopaedics-Sports medicine Center, Lexington (W.B.K.), Kentucky, U.S.A.

The authors report the following potential conflicts of interest or sources of funding: A.V.S. reports other from Flexion Therapeutics, Arthritis Foundation, AlloSource, Smith $\theta$ Nephew, Arthrex, Stryker, Medwest Associates, DJO, Wright Medical, and CDC Medical, outside the submitted work. Full ICMJE author disclosure forms are available for this article online, as supplementary material.

Received July 5, 2021; accepted December 1, 2021.

Address correspondence to Austin V. Stone, M.D., Ph.D., Division of Sports Medicine, Department of Orthopaedic Surgery and Sports Medicine, University of Kentucky, 740 S. Limestone, K401, Lexington, KY 40503. E-mail: austinvstonemd@gmail.com

(C) 2022 THE AUTHORS. Published by Elsevier Inc. on behalf of the Arthroscopy Association of North America. This is an open access article under the CC BY-NC-ND license (http://creativecommons.org/licenses/by-nc-nd/4.0/).

2666-061X/21965

https://doi.org/10.1016/j.asmr.2021.12.003 comprised $17 \%$ of all injuries from 2011 to $2016 .{ }^{1}$ More than $75 \%$ of those shoulder injuries occurred in pitchers. ${ }^{1}$ While multiple risk factors have been identified for upper-extremity injury in pitchers, particularly to the ulnar collateral ligament, ${ }^{2-9}$ risk factors for shoulder injury are not well documented ${ }^{5}$ despite their frequency.

Studies show a strong correlation between shoulder position kinematics in the pitching motion, control over pitch location (command), ${ }^{10}$ and upper-extremity injury. ${ }^{11-13}$ Alterations in these overhead kinematics are thought to increase injury risk. Investigations of risk for upper-extremity injury pertaining to shoulder positioning were performed in biomechanical studies. ${ }^{14}$ Glenohumeral internal rotational deficit, ${ }^{11}$ loss of shoulder rotational range of motion, and excessive glenohumeral horizontal abduction ${ }^{12}$ have been demonstrated to be risk factors for upper extremity injury in baseball players. ${ }^{13}$ Alterations in kinematics leading to injury have also been shown to occur in tennis players. ${ }^{15,16}$ In addition, performance parameters such as loss of command (alteration in pitch location) and decrease in pitch velocity have been thought to be early indicators of shoulder injury in pitchers ${ }^{17}$ 


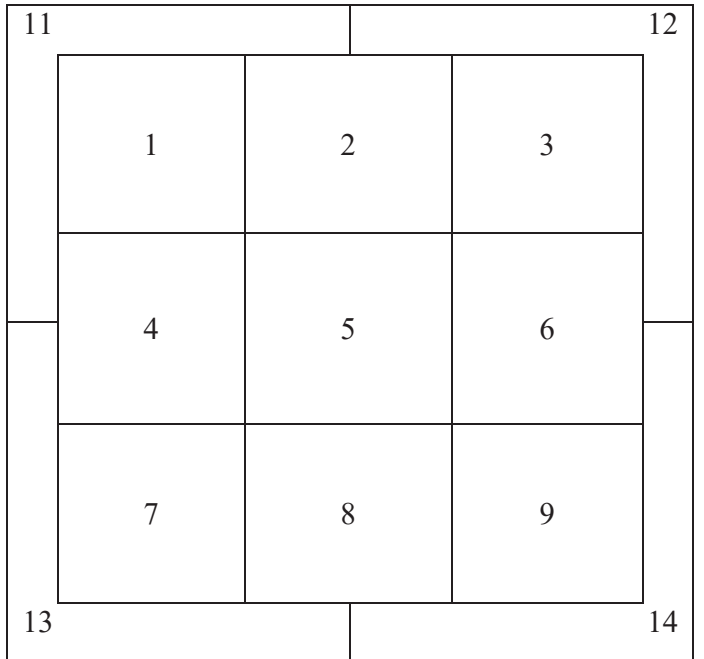

Fig 1. Game-Day Zones as defined by Statcast and Baseball Savant. The strike zone is presented as seen from the catcher's point of view. Zones 1-9 are within the regulation strike zone, zones 11-14 are outside of what would be considered a called strike. $^{24}$

and have been shown to be indicators of injury in tennis players. ${ }^{15,16}$ Glanzer et al. $^{10}$ identified that reducing the variability in shoulder position is associated with improved pitch location. The authors found significant predictors for pitch location include shoulder abduction and horizontal abduction at time of foot contact as well as shoulder external rotation and horizontal adduction at point of maximum shoulder external rotation. ${ }^{10}$ The authors concluded that decreases in variability in shoulder positioning at key points during the pitching motion may improve pitch accuracy. ${ }^{10}$ Other studies found that the most common errors in pitch accuracy were associated with shoulder fatigue $^{18}$ and were in the vertical direction. ${ }^{19}$

Pitch location is a critical component of success for a pitcher. The details of pitch placement have led to significant changes in strategy for pitchers in recent years based on advanced analytics, such as increasingly elevated 4-seam fastballs, increasing perceived velocity, and decreasing average exit velocity. ${ }^{20}$ Advanced pitch tracking technology is now available publicly via Baseball Savant, which uses Major League Baseball (MLB)'s Statcast data using multiple high-speed cameras and radar systems to report ball tracking metrics. ${ }^{21}$ The system is implemented by the MLB via Washington State University and carries specifications of spatial resolution of 0.03 inches/pixel, exposure times of $50 \mu \mathrm{s}$, with motion blur $<0.080$ inches. $^{22}$

The purpose of this study was to evaluate whether impending shoulder injury was associated with changes in pitch location or velocity immediately preceding injury. We hypothesized that pitchers would demonstrate changes in pitch location, specifically increases in pitches thrown high, toward the pitcher's arm-side, and out of the zone, between 1 year and 1 month before placement on the injured list (IL) for diagnosed shoulder injury.

\section{Methods}

The study was undertaken with a retrospective case series design. Shoulder injuries were first identified using transaction reports from mlb.com. ${ }^{23}$ Shoulder injuries from 2015 to 2020 were included for all pitchers who were placed on the IL with a chief complaint including the word "shoulder" on his throwing side. Placement on the IL requires a medical diagnosis after a physician's determination that the player is unable to play. ${ }^{24}$ The reason for being placed on the IL and the date of placement on the IL was collected from MLB transaction reports ${ }^{23}$ for each pitcher.

For each pitcher, the number of pitches recorded in each Game Day Zone (Fig 1) ${ }^{24}$ was recorded from Baseball Savant ${ }^{21}$ for 1 month before placement on the IL (Injury time frame) and for the season preceding placement on the IL (Baseline time frame). The horizontal axis was reversed for left-handed pitchers such that the arm side of all pitchers were collected in zones 1-3, 11, and 13, as seen in Figure 1. Pitches collected included the 4-seam fastball, slider, curveball, and change-up. Average 4-seam fastball velocity also was collected in each time frame. The curveball and slider data were pooled into one breaking ball category based on biomechanical similarity. ${ }^{25}$ The proportions of pitches in each Game Day Zones were calculated for each pitch type for those who qualified (Fig 2).

A minimum of 55 pitches of each pitch type was used for inclusion based on an estimate of the stabilization rate of pitch location distribution which was calculated using the known stabilization rates of common baseball

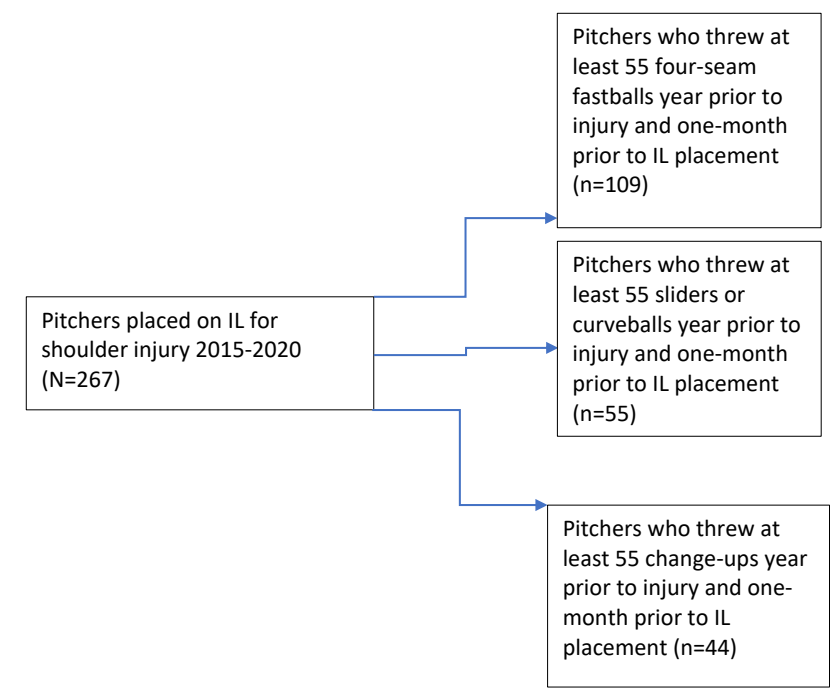

Fig 2. Inclusion diagram for pitchers included in case series design. (IL, injured list.) 
Table 1. Shoulder Injury Demographics

\begin{tabular}{lc}
\hline \multicolumn{1}{c}{ Injury } & Number $(\%)$ \\
\hline Inflammation & $89(33)$ \\
Strain/sprain & $69(26)$ \\
Impingement & $25(9.4)$ \\
Tendinitis & $22(8.2)$ \\
Soreness & $18(6.7)$ \\
Fatigue ( & $12(4.5)$ \\
Surgery & $6(2.2)$ \\
Tightness & $6(2.2)$ \\
Discomfort & $6(2.2)$ \\
Bursitis (\%) & $4(1.5)$ \\
Torn labrum & $1(0.4)$ \\
Scapular stress injury & $1(0.4)$ \\
Thoracic outlet syndrome & $1(0.4)$ \\
Contusion & $1(0.4)$ \\
Subluxation & $1(0.4)$ \\
Stiffness & $1(0.4)$ \\
N/a & $4(1.5)$ \\
\hline N/a, not available.
\end{tabular}

N/a, not available.

metrics such as strikeout rate and walk rate. ${ }^{26}$ The evaluation of pitch command relied on the new ability to assess individual pitch placement rather than plate appearance outcomes such as walk rate or strikeout rate. The number of pitches was selected based on the following factors. The stabilization rates of such statistics are intrinsically related to their frequency of occurrence in which walk rates, around $8 \%$ in the present era, ${ }^{27}$ stabilize after 170 batters faced and strikeout rates, approximately $20 \%$ in the present era, ${ }^{26}$ and stabilize after 70 batters faced. ${ }^{26}$ Collecting individual pitch locations multiplies the data available for collection per batter. While determining strikeout incidence takes an average of 5 pitches $^{28}$ for each data point, the evaluation of each pitch individual allows for the collection of 5 data points for the same plate appearance, thus enabling a more detailed look at the command of a pitcher and allowing for small but significant differences to be detected.

\section{Statistical Analysis}

Game Day Zones were consolidated into comparisons of high (Zones 1, 2, 3, 11, and 12) versus low (Zones 7, $8,9,13$, and 14), arm-side (Zones 1, 4, 7, 11, and 13) versus opposite-side (Zones 3, 7, 9, 12, and 14), and in zone (Zones 1-9) versus out of zone (Zones 11-14). Repeated measures analysis of variance (ANOVA) was performed to determine significant differences between baseline and injury time frames in pitch location and 4seam fastball velocity. The linear model using timeframe as the sole independent variable was compared to an analysis of covariance model (ANCOVA) including age, position (starter vs reliever), and total pitches thrown in the month preceding injury and the season preceding injury as covariates with an ANOVA to determine if the covariates had a significant confounding effect. Normality was assessed visually using QQ plots and density plots, which revealed each variable distribution to be approximately normal. ${ }^{29}$ The assumption of sphericity was checked using Mauchly's test of sphericity. To control for potential league-wide strategic changes in more recent seasons regarding throwing elevated 4-seam fastballs, a repeatedmeasures ANCOVA was performed for the high versus low 4 -seam fastball analysis, which included year of season as a covariate. $\mathrm{R}$ software, version 4.0.2 (R Foundation for Statistical Computing, Vienna, Austria) was used for data analysis. ${ }^{30}$ Significance was set at $P<.05$.

\section{Results}

A total of 267 pitcher injury list placements due to a shoulder diagnosis were identified from 2015 to 2020. The most common reason for being placed on the IL was inflammation $(33.3 \%)$ followed by strain/sprain $(25.8 \%)$, impingement $(9.4 \%)$, and tendinitis $(8.2 \%)$. Additional reasons for IL placement included soreness, fatigue, surgery, tightness, discomfort, bursitis, torn labrum, scapular stress injury, thoracic outlet syndrome, contusion, subluxation, and stiffness. Four players $(1.5 \%)$ did not have a reason for being placed on the IL more specific than shoulder injury (Table 1). Of the 155 players with a minimum amount of data for analysis in at least 1 pitch, the vast majority $(\mathrm{n}=148$, $95.5 \%)$ were 10 - to 15 -day IL stints, and the minority ( $\mathrm{n}=7,4.5 \%)$ were 60 -day IL placements.

There was no significant difference in 4-seam fastball velocity or proportion of arm-side 4-seam fastballs thrown 1 month before injury (Table 2). There were significantly more elevated 4-seam fastballs thrown 1 month before being placed on the IL for a shoulder injury compared with baseline $(45.9 \%$ vs $42.4 \%, P=$ .008 , Table 2, Fig 3). There was also a significantly greater proportion of 4-seam fastballs thrown out of the strike zone 1 month before placement on the IL $(48.5 \%$ vs $46.5 \%, P=.011$, Table 2 , Fig 3$)$. The ANCOVA with age, position, and total pitches thrown was shown to be insignificantly different from the ANOVA models for elevated four-seam fastballs and four-seam fastballs out of the strike zone $(P=.514$ and $P=.668$, respectively). There were no significant differences in location of

Table 2. Four-Seam Fastball Results, $n=109$; There Were Significantly Increases in High Four-Seam Fastballs and FourSeam Fastballs Thrown Outside of the Zone

\begin{tabular}{lccc}
\hline \multicolumn{1}{c}{ Metric } & Baseline & Injury & $P$ Value \\
\hline Velocity, mph (SD) & $93.7(2.4)$ & $93.2(2.5)$ & .190 \\
High location, \% (SD) & $42.4(9.0)$ & $45.9(10.4)$ & $\mathbf{. 0 0 8}$ \\
Arm-side location, \% (SD) & $38.0(8.4)$ & $37.3(10.3)$ & .613 \\
Out of zone, \% (SD) & $46.5(5.2)$ & $48.5(6.3)$ & $\mathbf{. 0 1 1}$ \\
\hline
\end{tabular}

SD, standard deviation. 


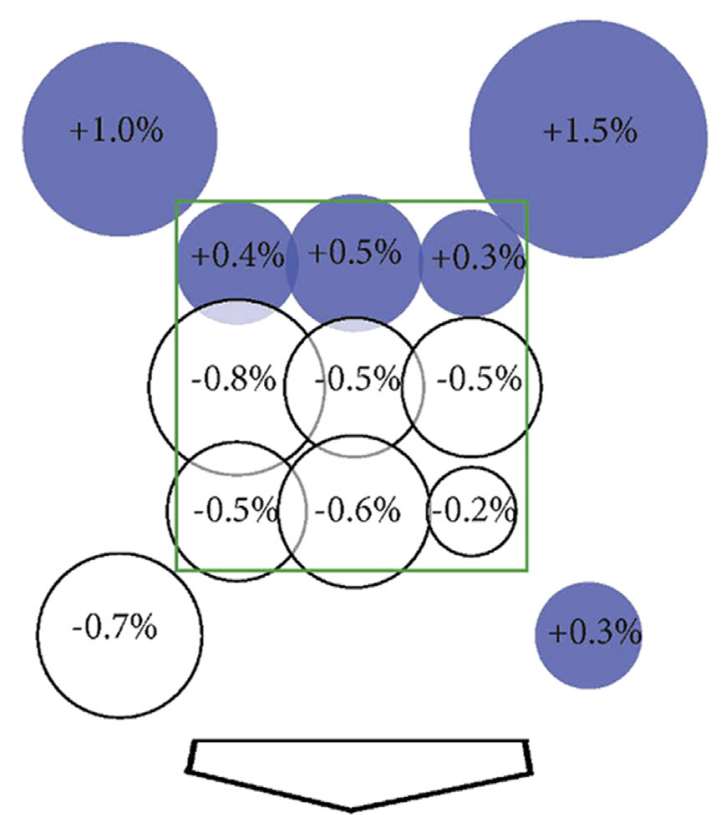

Fig 3. Four-seam fastball location changes. (Injury \% Baseline \%), Blue denotes an increase in frequency, white denotes a decrease in frequency, and the strike zone is represented by the green box. Pitches in the high zones (GameDay Zones 1-3, 11, 12; $P=.008$ ) and out of the strike zone (Zones 11, 12, 13, 14; $P=.011$ ) were more frequent.

breaking balls or change-ups 1 month prior to placement on the IL (Tables 3 and 4).

In an ANCOVA, season-year was found to be significantly associated with increased high 4 -seam fastballs $(+2.2 \%$ per year, $P<.001)$; however, being in the time frame 1 month before IL placement was found to be independently associated with a significant increase in high 4 -seam fastballs $(+3.7 \%$ in the injury time frame, $P=.006)$.

\section{Discussion}

The principal finding of this study is that pitch location changed in elevated 4 -seam fastballs and fastballs thrown out of the strike zone before pitcher's placement on the injury list for a shoulder injury. Age, total pitches thrown, and position (starter vs reliever) were not found to be significant confounders in this analysis. The increased frequency of elevated 4-seam fastballs occurred independently of the season. Interestingly, 4-seam fastball velocity did not change compared with baseline one-month prior to IL placement.

Table 3. Breaking Ball Results, $\mathrm{n}=55$; No Significant Differences Were Found in Breaking Ball Location at Baseline and Immediately Preceding Injury

\begin{tabular}{lllc}
\hline \multicolumn{1}{c}{ Metric } & \multicolumn{1}{c}{ Baseline } & \multicolumn{1}{c}{ Injury } & $P$ Value \\
\hline High location, \% (SD) & $17.6(6.7)$ & $16.3(7.2)$ & .555 \\
Arm-side location, \% (SD) & $26.1(11.6)$ & $24.7(11.0)$ & .670 \\
Out of zone, \% (SD) & $57.0(9.7)$ & $57.9(9.0)$ & .485 \\
\hline SD, standard deviation. & &
\end{tabular}

Table 4. Change-Up Results, $n=44$; No Significant Differences Were Found in Change-Up Location at Baseline and Immediately Preceding Injury

\begin{tabular}{lllc}
\hline \multicolumn{1}{c}{ Metric } & \multicolumn{1}{c}{ Baseline } & \multicolumn{1}{c}{ Injury } & $P$ Value \\
\hline High location, \% (SD) & $17.2(7.7)$ & $16.5(8.4)$ & 0.729 \\
Arm-side location, \% (SD) & $62.5(10.4)$ & $60.8(12.9)$ & 0.488 \\
Out of zone, \% (SD) & $59.0(8.2)$ & $58.3(9.2)$ & 0.718 \\
\hline SD, standard deviation. & & &
\end{tabular}

SD, standard deviation.

This study adds to the current literature in several important ways. It adds credence to the anecdotal perception that loss of control may indicate early stages of throwing injury. ${ }^{17}$ As pitching command issues have been a portion of recording the subjective history of present illness from pitchers, these data supply objective confirmation of the notion that command is one of the performance changes associated with injury. While the absolute difference recorded was small, the ability to record the location of individual pitches along with the frequency of use of the four-seam fastball allowed for a detection of a statistically significant difference which corresponds with the subjective perception of pitchers and coaches. ${ }^{17}$ As such, inquiring about loss of command or velocity are recommended portions of the history to collect when evaluating a potentially injured pitcher. ${ }^{17}$ It is important to note that high 4 -seam fastballs increased immediately preceding injury even after considering the season year as elevated 4-seam fastballs have increased in frequency in recent years. ${ }^{20}$ This suggests that the increase in elevated 4-seam fastballs was not a conscious effort by the pitcher. This is further supported by an increase in 4-seam fastballs thrown outside of the strike zone.

This descriptive study cannot provide information regarding the exact relationship between a shoulder injury and the change in pitch location. The command changes may be explained by early shoulder injury leading to altered kinematics in the pitching motion, or the altered kinematics may increase risk for future injury and result in the altered pitch location and command. Another consideration is the loss of proprioception that occurs with fatigue due to exercise. ${ }^{31-34}$ As positioning of the shoulder has been shown to be critical to command, particularly in the 4-seam fastball, this rationale is consistent with recent biomechanical evaluations of pitching. ${ }^{10}$

A concept that links these 2 possible mechanisms is that of the launch window, ${ }^{19}$ a finite time and arm position at which ball release results in optimum ball speed and pitch accuracy. Errors in pitch accuracy were found to be almost always errors in vertical accuracy and were attributed to occur from either release errors (timing of ball release from the fingers) or from alterations of arm path (altered kinematics in the body segments proximal to the hand). ${ }^{15,16}$ Relating this 
concept to the findings of this study, the altered performance variable, pitch location, which was in the vertical direction in confirmation of the expectation from the original launch window study, would result from the altered launch window created by the arm path error, which could result from either the injury producing the altered kinematics or the altered kinematics producing the injury. The clinical implications are that pitch location should be monitored closely, and if documented, comprehensive evaluation for all kinetic chain sources of altered kinematics, especially scapular and shoulder motion, should be performed.

Our results are consistent with biomechanical investigations of other overhead throwing athletes such as tennis players. Martin et al. ${ }^{15}$ showed significant differences in the kinetics and energy transfer efficiency in the tennis serves of players who went on to develop upperextremity injury 2 years following motion capture compared to those without. The authors concluded that maximizing performance and minimizing injury risk were not mutually exclusive as players who did not develop upper-extremity injury had the more efficient proximodistal kinetic energy transfer and higher serve velocity compared to those who developed injury. ${ }^{15}$ Similarly, Martin et al. ${ }^{16}$ showed that more efficient serve kinetics were associated with not only greater performance level attained on average by tennis players but also decreased shoulder inferior force, anterior force, and abduction torque. ${ }^{16}$ These findings further suggest that maximizing kinetic energy transfer and body kinematics improves performance while decreasing risk for injury. These results in high-level tennis athletes suggest that a change in kinematics led to upper extremity injury. A common underlying mechanism between prior studies and the current study may be that loss of proper kinematics leads to the injury rather than the injury preceding poor kinematics. This theory is supported by the established association between muscular fatigue and loss of proprioception. ${ }^{31}$ While we cannot definitively demonstrate that early shoulder injury led to kinematic change ultimately leading to loss of command, our data suggest that loss of 4-seam fastball command may be an early indicator of either altered body kinematics or impending shoulder injury. Pitch location should be closely monitored, and if alterations are found, then an all-kinetic chain sources of altered kinematics should be evaluated, especially scapular and shoulder motion,

A second contribution our analysis makes to the current understanding of early shoulder injuries in overhead throwing athletes is the lack of statistically significant changes in 4-seam fastball velocity immediately before IL placement among the 109 pitchers included. While velocity changes remain an important part of the history of overhead throwing complaints, ${ }^{17}$ our analysis suggests that velocity decreases are not a sensitive component of injury evaluation. There are multiple methods of proximal kinetic chain compensations to maintain arm velocity ${ }^{35,36}$; however, these compensations may not result in optimal arm path motion and kinematics. Suboptimal arm path motion and kinematics increase distal loads, ${ }^{35,36}$ alter the optimum launch window, and result in pitch location errors. Also, pitch location errors were found to be associated with shoulder alterations. ${ }^{18}$ Similar compensatory findings also are found in tennis players with a history of shoulder problems who try to preserve shoulder integrity during the serve and compete by altering their humerothoracic and scapulothoracic orientations. ${ }^{37}$ In other words, maintenance of average pitch velocity does not preclude the presence of altered kinematics or shoulder injury.

\section{Limitations}

The present manuscript carries several limitations. The specific population of MLB pitchers is not necessarily generalizable to all baseball pitchers or overhead throwing athletes. Publicly accessible databases limit the resolution of reported shoulder injuries, so the experimental group injuries are heterogeneous. In addition, the study design did not allow the determination of specific injuries or specific alterations in kinematics that may have been associated with injury or the altered pitch location. More so, the case series design cannot offer information on the specificity of location changes in identifying shoulder injury. In other words, more factors may lead to pitching location changes than shoulder injury that were not included in the analysis.

\section{Conclusions}

Pitchers threw more elevated 4-seam fastballs and out-of-zone 4-seam fastballs in the month before IL placement for shoulder injury. These findings suggest that a loss of 4-seam fastball command decreases with impending shoulder injury.

\section{References}

1. Fares MY, Fares J, Baydoun H, Fares Y. Prevalence and patterns of shoulder injuries in Major League Baseball. Phys Sportsmed 2020;48:63-67.

2. Camp CL, Zajac JM, Pearson DB, et al. Decreased shoulder external rotation and flexion are greater predictors of injury than internal rotation deficits: Analysis of 132 pitcher-seasons in professional baseball. Arthroscopy 2017;33:1629-1636.

3. DeFroda SF, Kriz PK, Hall AM, Zurakowski D, Fadale PD. Risk stratification for ulnar collateral ligament injury in Major League Baseball players: A retrospective study from 2007 to 2014. Orthop J Sports Med 2016;4: 2325967115627126.

4. Erickson BJ, Chalmers PN, Zajac J, et al. Do professional baseball players with a higher valgus carrying angle have an increased risk of shoulder and elbow injuries? Orthop J Sports Med 2019;7:2325967119866734. 
5. Chalmers PN, Erickson BJ, D’Angelo J, Ma K, Romeo AA. Epidemiology of shoulder surgery among professional baseball players. Am J Sports Med 2019;47:1068-1073.

6. Keller RA, Marshall NE, Guest JM, Okoroha KR, Jung EK, Moutzouros V. Major League Baseball pitch velocity and pitch type associated with risk of ulnar collateral ligament injury. J Shoulder Elbow Surg 2016;25:671-675.

7. Portney DA, Buchler LT, Lazaroff JM, Gryzlo SM, Saltzman MD. Influence of pitching release location on ulnar collateral ligament reconstruction risk among major league baseball pitchers. Orthop J Sports Med 2019;7: 2325967119826540

8. Chalmers PN, Erickson BJ, Ball B, Romeo AA, Verma NN. Fastball pitch velocity helps predict ulnar collateral ligament reconstruction in major league baseball pitchers. Am J Sports Med 2016;44:2130-2135.

9. Prodromo J, Patel N, Kumar N, Denehy K, Tabb LP, Tom J. Pitch characteristics before ulnar collateral ligament reconstruction in major league pitchers compared with age-matched controls. Orthop J Sports Med 2016;4: 2325967116653946.

10. Glanzer JA, Diffendaffer AZ, Slowik JS, Drogosz M, Lo NJ, Fleisig GS. The relationship between variability in baseball pitching kinematics and consistency in pitch location. Sports Biomech 2021;20:879-886.

11. Burkhart SS, Morgan CD. The peel-back mechanism: Its role in producing and extending posterior type II SLAP lesions and its effect on SLAP repair rehabilitation. Arthroscopy 1998;14:637-640.

12. Mihata T, McGarry MH, Kinoshita M, Lee TQ. Excessive glenohumeral horizontal abduction as occurs during the late cocking phase of the throwing motion can be critical for internal impingement. Am J Sports Med 2010;38: 369-374.

13. Wilk KE, Macrina LC, Fleisig GS, et al. Deficits in glenohumeral passive range of motion increase risk of elbow injury in professional baseball pitchers: A prospective study. Am J Sports Med 2014;42:2075-2081.

14. Chalmers PN, Wimmer MA, Verma NN, et al. The relationship between pitching mechanics and injury: A review of current concepts. Sports Health 2017;9: 216-221.

15. Martin C, Bideau B, Bideau N, Nicolas G, Delamarche P, Kulpa R. Energy flow analysis during the tennis serve: Comparison between injured and noninjured tennis players. Am J Sports Med 2014;42:2751-2760.

16. Martin C, Bideau B, Ropars M, Delamarche P, Kulpa R. Upper limb joint kinetic analysis during tennis serve: Assessment of competitive level on efficiency and injury risks. Scand J Med Sci Sports 2014;24:700-707.

17. Reinold MM, Gill TJ. Current concepts in the evaluation and treatment of the shoulder in overhead-throwing athletes, part 1: Physical characteristics and clinical examination. Sports Health 2010;2:39-50.

18. Freeston J, Adams R, Ferdinands RE, Rooney K. Indicators of throwing arm fatigue in elite adolescent male baseball players: A randomized crossover trial. J Strength Cond Res 2014;28:2115-2120.
19. Freeston J, Ferdinands RE, Rooney K. The launch window hypothesis and the speed-accuracy trade-off in baseball throwing. Percept Mot Skills 2015;121:135-148.

20. Sawchik T. Baseball's stop staffs have come around on the high-spin fastball. Fivethirtyeight. https://fivethirtyeight. com/features/baseballs-top-staffs-have-come-around-onthe-high-spin-fastball/. Accessed November 3, 2021.

21. Willman D. Baseball Savant. Major League Baseball; 2015. Baseballsavant.mlb.com. Accessed March 1, 2021.

22. Nunnally C. MLB Ground Truth Testing. Vol 2020: MLB Advanced Media 2019. https://technology.mlblogs.com/ mlb-ground-truth-testing-ec87c73450b9. Accessed March 1, 2021.

23. MajorLeagueBaseballTransactions. MLB Transactions. Vol 2020. New York; 1995. http://mlb.mlb.com/mlb/ transactions/. Accessed March 1, 2021.

24. MLBMiscellany. MLB Miscellany: Rules, regulation and statistics. Vol 2020. http://mlb.mlb.com/mlb/official_info/ about_mlb/rules_regulations.jsp. Accessed March 1, 2021.

25. Fleisig GS, Kingsley DS, Loftice JW, et al. Kinetic comparison among the fastball, curveball, change-up, and slider in collegiate baseball pitchers. Am J Sports Med 2006;34:423-430.

26. Slowinski P. Sample Size. 2010. Vol 2021 . library.fangraphs. com/principles/sample-size/. Accessed March 1, 2021.

27. Appelman D. Fangraphs.com. Arlington, VA: Fangraphs Inc., 2005.

28. Arthur R. We're Seeing More Strikeouts, But It Takes Many More Pitches to Get Them. 2015. Vol 2021. fivethirtyeight. com/features/were-seeing-more-strikeouts-but-what-doesthat-tell-us/. Accessed March 1, 2021.

29. Field A. Discovering statistics using SPSS, 3rd ed. Los Angeles, CA: SAGE Publications, 2009.

30. R Development Core Team. R. A language and environment for statistical computing. Vienna, Austria: R Foundation for Statistical Computing, 2010.

31. Proske U. Exercise, fatigue and proprioception: A retrospective. Exp Brain Res 2019;237:2447-2459.

32. Tripp BL, Yochem EM, Uhl TL. Functional fatigue and upper extremity sensorimotor system acuity in baseball athletes. J Athl Train 2007;42:90-98.

33. Tripp BL, Yochem EM, Uhl TL. Recovery of upper extremity sensorimotor system acuity in baseball athletes after a throwing-fatigue protocol. J Athl Train 2007;42: 452-457.

34. Tripp BL, Uhl TL, Mattacola CG, Srinivasan C, Shapiro R. Functional multijoint position reproduction acuity in overhead-throwing athletes. J Athl Train 2006;41:146-153.

35. Kibler WB. Biomechanical analysis of the shoulder during tennis activities. Clin Sports Med 1995;14:79-85.

36. Elliott B, Fleisig G, Nicholls R, Escamilia R. Technique effects on upper limb loading in the tennis serve. J Sci Med Sport 2003;6:76-87.

37. Gillet B, Begon M, Diger M, Berger-Vachon C, Rogowski I. Alterations in scapulothoracic and humerothoracic kinematics during the tennis serve in adolescent players with a history of shoulder problems. Sports Biomech 2021;20:165-177. 